\title{
Non-factorizable contributions to $\overline{B_{d}^{0}} \rightarrow D_{s}^{(*)} \overline{D_{s}^{(*)}}$
}

\author{
J.O. $\operatorname{Eeg}^{\mathrm{a} *}$, S. Fajfer ${ }^{\mathrm{bc}} \dagger$, A. Hiorth ${ }^{\mathrm{d}}$ and A. Prapotnik ${ }^{\mathrm{b}}$ \\ a Department of Physics, University of Oslo, P.O. Box 1048 Blindern, N-0316 Oslo, Norway \\ bJ. Stefan Institute, Jamova 39, SI-1000 Ljubljana, Slovenia \\ ${ }^{c}$ Physics Department, University of Ljubljana, Jadranska 19, SI-1000 Ljubljana, Slovenia \\ dRF-Rogaland Research, P.O.Box 8046, N-4068 Stavanger, Norway
}

It is pointed out that decays of the type $B \rightarrow D \bar{D}$ have no factorizable contributions, unless at least one of the charmed mesons in the final state is a vector meson. The dominant contributions to the decay amplitudes arise from chiral loop contributions and tree level amplitudes generated by soft gluon emissions forming a gluon condensate. We predict that the branching ratios for the processes $\bar{B}^{0} \rightarrow D_{s}^{+} D_{s}^{-}, \bar{B}^{0} \rightarrow D_{s}^{+*} D_{s}^{-}$and $\bar{B}^{0} \rightarrow$ $D_{s}^{+} D_{s}^{-*}$ are all of order $(3-4) \times 10^{-4}$, while $\bar{B}^{0} \rightarrow D_{s}^{+*} D_{s}^{-*}$ has a branching ratio 5 to 10 times bigger. We emphasize that the branching ratios are sensitive to $1 / m_{c}$ corrections.

\section{INTRODUCTION}

Within the standard approach for non-leptonic decays one constructs an effective Lagrangian in terms of (mainly) four quark operators multiplied with Wilson coefficients. The simple and naive assumption that the matrix elements of the four quark operators factorize as the product of two current matrix elements can be shown to be valid in the strict limit $N_{c} \rightarrow \infty$, where $N_{c}$ is the number of colors. In the treatment of $B$-meson decays with energy release of the order of the $b$ quark mass, usually the factorization assumption or QCD factorization has been used. However, for decays where the energy release is of order $1 \mathrm{GeV}$, QCD factorization is not expected to hold. Here we discuss non-factorizable contributions to the decay modes $\overline{B_{d}^{0}} \rightarrow D_{s}^{(*)} \overline{D_{s}^{(*)}}$, where $D_{s}^{(*)}$ is a pseudoscalar or a vector meson. At quark level such decays occur through the annihilation mechanism $b \bar{q} \rightarrow c \bar{c}$, where $q=d, s$ respectively. How-

\footnotetext{
*Presented by J.O.Eeg. Supported in part by the Norwegian research council and by the European Union RTN network, Contract No. HPRN-CT-2002-00311 (EURIDICE).

${ }^{\dagger}$ Supported in part by the Ministry of Education, Science and Sport of the Republic of Slovenia.
}

ever, within the factorized limit the annihilation mechanism will give a vanishing amplitude due to current conservation, unless one or two of the $D$-mesons in the final state are vectors. These contributions are proportional to a numerically non-favored Wilson coefficient.

In contrast, the typical factorized decay modes which proceed through the spectator mechanism, say $\overline{B^{0}} \rightarrow D^{+} D_{s}^{-}$, are proportional to the numerically favored Wilson coefficient. In our approach 1], the non-factorizable contributions are coming from the chiral loops and from tree level amplitudes generated by soft gluon emision forming a gluon condensate. The gluon condensate contributions can be calculated within a recently developed Heavy Light Chiral Quark Model (HL $\chi$ QM) 2]. This model has been applied to processes involving $B$-mesons in [23]. Both the chiral loop contributions and the gluon condensate contributions are proportional to the numerically favorable Wilson coefficient. 


\section{FRAMEWORK}

\subsection{Effective Lagrangian at quark level}

The effective Lagrangian at quark level reads:

$\mathcal{L}_{W}=-\frac{G_{F}}{\sqrt{2}} V_{c b} V_{c q}^{*} \sum_{i} a_{i}(\mu) Q_{i}(\mu)$,

where $q=d, s$ and $a_{i}(\mu)$ are Wilson coefficients that carry all information of the short distance physics above the renormalization scale $\mu$. The matrix elements of $Q_{i}(\mu)$ contain all non-perturbative, long distance physics below $\mu$. Within Heavy Quark Effective Theory (HQEFT) the effective non-leptonic Lagrangian $\mathcal{L}_{W}$ can be evolved down to the scale $\mu=\Lambda_{\chi} \simeq 1 \mathrm{GeV}$, and below $\mu=m_{c}$ the Wilson coefficients $a_{i}$ are complex [4.

The numerically relevant operators in our case are

$Q_{1}=4\left(\bar{q}_{L} \gamma^{\mu} b_{L}\right)\left(\bar{c}_{L} \gamma_{\mu} c_{L}\right)$,

$Q_{2}=4\left(\bar{c}_{L} \gamma^{\mu} b_{L}\right)\left(\bar{q}_{L} \gamma_{\mu} c_{L}\right)$,

where $L$ denotes a left-handed particle. At $\mu=\Lambda_{\chi}$, which by construction is the matching scale within our approach 123, one finds $a_{1} \simeq-0.35-0.07 i$ and $a_{2} \simeq 1.29+0.08 i$. In the next section we will see how the currents in the operators in (2) are bosonized.

In order to obtain all matrix elements of the Lagrangian (1) we need the Fierz transformed version of the operators in (2). To find these, we use the relation:

$\delta_{i j} \delta_{l n}=\frac{1}{N_{c}} \delta_{i n} \delta_{l j}+2 t_{i n}^{a} t_{l j}^{a}$,

where $i, j, k$ and $n$ are color indices running from 1 to 3 and $a$ is a color octet index. One obtains

$Q_{1}^{F}=\frac{1}{N_{C}} Q_{2}+\widetilde{Q}_{2}, \quad Q_{2}^{F}=\frac{1}{N_{C}} Q_{1}+\widetilde{Q}_{1}$,

where the superscript $F$ means "Fierzed", and

$\widetilde{Q_{1}}=4\left(\bar{q}_{L} \gamma^{\mu} t^{a} b_{L}\right)\left(\bar{c}_{L} \gamma_{\mu} t^{a} c_{L}\right)$,

$\widetilde{Q_{2}}=4\left(\bar{c}_{L} \gamma^{\mu} t^{a} b_{L}\right)\left(\bar{q}_{L} \gamma_{\mu} t^{a} c_{L}\right)$,

where $t^{a}$ denotes the color matrices. These expressions are used to obtain the gluon condensate contributions.

\subsection{Heavy light chiral perturbation theory} The Heavy Quark Effective Theory (HQEFT) Lagrangian is:

$\mathcal{L}_{H Q E F T}= \pm \overline{Q_{v}^{( \pm)}} i v \cdot D Q_{v}^{( \pm)}+\mathcal{O}\left(m_{Q}^{-1}\right)$,

where $Q_{v}^{(+)}(x)$ is a (reduced) heavy quark field (b or $c$ in our case) with velocity $v$, and $Q_{v}^{(-)}(x)$ is the field of a heavy anti-quark ( $\bar{c}$ in our case). Furthermore, $m_{Q}$ is the heavy quark mass, and $D_{\mu}$ is the covariant derivative containing the gluon field.

After integrating out the heavy and light quarks, the effective Lagrangian up to $\mathcal{O}\left(m_{Q}^{-1}\right)$ can be written as a kinetic term plus a term describing the chiral interaction between heavy and light mesons [2]:

$\mathcal{L}_{\chi}=-g_{\mathcal{A}} \operatorname{Tr}\left[\overline{H_{a}^{( \pm)}} H_{b}^{( \pm)} \gamma_{\mu} \gamma_{5} \mathcal{A}_{b a}^{\mu}\right]$,

where $H_{a}^{( \pm)}$is the heavy meson field containing a spin zero and a spin one boson:

$H_{a}^{( \pm)}=P_{ \pm}\left(P_{a \mu}^{( \pm)} \gamma^{\mu}-i P_{a 5}^{( \pm)} \gamma_{5}\right)$.

Here $a, b$ are flavor indices and $P_{ \pm}=(1 \pm \gamma \cdot v) / 2$ are projecting operators. The axial vector field $\mathcal{A}_{\mu}$ in (7) is defined as:

$\mathcal{A}_{\mu}=-\frac{i}{2}\left(\xi^{\dagger} \partial_{\mu} \xi-\xi \partial_{\mu} \xi^{\dagger}\right)$,

where $\xi \equiv \exp [i(\Pi / f)]$. Moreover, $f$ is the bare pion coupling and $\Pi$ is a 3 by 3 matrix which contains the Goldstone bosons $\pi, K, \eta$ in the standard way. The axial chiral coupling $g_{\mathcal{A}}$ is $\simeq 0.6$.

Based on the symmetry of HQEFT, we obtain the bosonized currents. For a decay of the $b \bar{q}$ system (see Fig. 10 we have [2]:

$\overline{q_{L}} \gamma^{\mu} Q_{v_{b}}^{(+)} \longrightarrow \frac{\alpha_{H}}{2} \operatorname{Tr}\left[\xi^{\dagger} \gamma^{\alpha} L H_{b}^{(+)}\right]$,

where (up to QCD and $1 / m_{Q}$ corrections [2]) $\alpha_{H}=f_{H} \sqrt{m_{H}}$ for $H=B, D$. Further, $Q_{v_{b}}^{(+)}$ is the heavy $b$-quark field, $v_{b}$ is its velocity, and $H_{b}^{(+)}$is the corresponding heavy meson field. For the $W$-boson materializing to a $\bar{D}$, the bosonized current $\overline{q_{L}} \gamma^{\mu} Q_{v_{\bar{c}}}^{(-)}$is given by (10) but with $H_{b}^{(+)}$ replaced by $H_{\bar{c}}^{(-)}$representing the $\bar{D}$ meson. $v_{\bar{c}}$ is the velocity of the heavy $\bar{c}$ quark. 


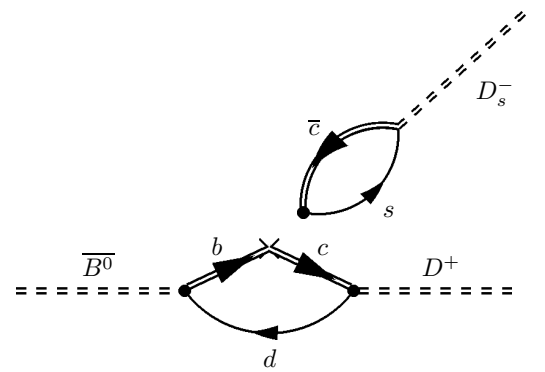

Figure 1. Factorized contribution for $\overline{B^{0}} \rightarrow D^{+} D_{s}^{-}$ through the spectator mechanism, which does not exist for decay mode $\overline{B^{0}} \rightarrow D_{s}^{+} D_{s}^{-}$. There are similar diagrams with vector mesons.

The bosonized $b \rightarrow c$ transition current in Fig. 11 is given by

$\overline{Q_{v_{b}}^{(+)}} \gamma^{\mu} L Q_{v_{c}}^{(+)} \longrightarrow-\zeta(\omega) \operatorname{Tr}\left[\overline{H_{c}^{(+)}} \gamma^{\alpha} L H_{b}^{(+)}\right]$

where $\zeta(\omega)$ is the Isgur-Wise function for the $\bar{B} \rightarrow$ $D$ - transition, and $v_{c}$ is the velocity of the heavy $c$-quark. Furthermore, $\omega \equiv v_{b} \cdot v_{c}=v_{b} \cdot v_{\bar{c}}=$ $M_{B} /\left(2 M_{D}\right)$.

For the weak current for $D \bar{D}$ production (corresponding to the factorizable annihilation mechanism in Fig. 2), the current $\overline{Q_{v_{c}}^{(+)}} \gamma^{\mu} L Q_{v_{\bar{c}}}^{(-)}$is given by (11) with $H_{b}^{(+)}$replaced by $H_{\bar{c}}^{(-)}$, and $\zeta(\omega)$ is replaced by $\zeta(-\lambda)$, where $\lambda=v_{\bar{c}} \cdot v_{c}=$ $\left[M_{B}^{2} /\left(2 M_{D}^{2}\right)-1\right]$. Note that $\zeta(-\lambda)$ is a complex function which is less known than $\zeta(\omega)$.

The factorized contributions for the spectator and annihilation diagrams are shown in Figs. 11, 2 The first diagram do not give any (direct) contributions to the class of processes we consider, but is still important because it is the basis of our chiral loops, visualized in Fig. 3

The chiral loop amplitudes are of order $\left(g_{\mathcal{A}} m_{K} / 4 \pi f\right)^{2}$ compared to typical factorizable amplitudes in processes where these exist.

\subsection{The HL $\chi \mathrm{QM}$}

The HL $\chi$ QM Lagrangian is

$\mathcal{L}_{\mathrm{HL} \chi \mathrm{QM}}=\mathcal{L}_{H Q E F T}+\mathcal{L}_{\chi Q M}+\mathcal{L}_{\text {Int }}$.

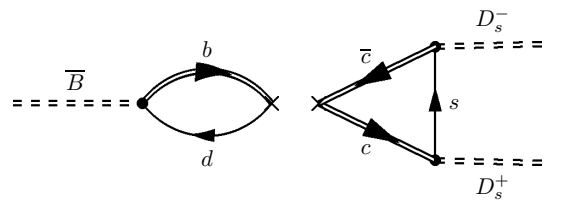

Figure 2. Factorized contribution for $\overline{B^{0}} \rightarrow D_{s}^{+} D_{s}^{-}$ through the annihilation mechanism, which give zero contributions if both $D_{s}^{+}$and $D_{s}^{-}$are pseudoscalars.
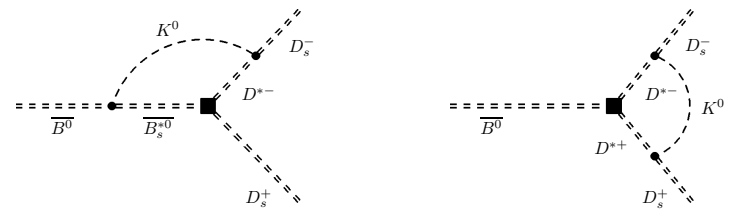

Figure 3. Non-factorizable chiral loops for $\overline{B^{0}} \rightarrow$ $D_{s}^{+} D_{s}^{-}$. There are similar diagrams for vector mesons in the final state.

The first term is given in (6) and the second term is described by the Chiral Quark Model of the light sector involving interactions between quarks and (Goldstone) mesons:

$\mathcal{L}_{\chi Q M}=\bar{\chi}\left[\gamma^{\mu}\left(i \mathcal{D}_{\mu}+\gamma_{5} \mathcal{A}_{\mu}\right)-m\right] \chi$.

Here $m=(230 \pm 20) \mathrm{MeV}$ is the $S U(3)$ invariant constituent light quark mass, and $\chi$ is the flavor rotated quark field given by $\chi_{L}=\xi^{\dagger} q_{L}$ and $\chi_{R}=\xi q_{R}$, where $q^{T}=(u, d, s)$ is the light quark field. The covariant derivative $\mathcal{D}_{\mu}$ contains the soft gluon field forming the gluon condensates (besides some chiral interactions) 235].

The interaction between heavy meson fields and quarks is described by [2]

$\mathcal{L}_{\text {Int }}=-G_{H}\left[\bar{\chi}_{a} \overline{H_{a}^{( \pm)}} Q_{v}^{( \pm)}+\overline{Q_{v}^{( \pm)}} H_{a}^{( \pm)} \chi_{a}\right]$

where the coupling constant $G_{H}=\sqrt{2 m \rho} / f$, and $\rho$ is a hadronic parameter depending on $m$ (nu- 


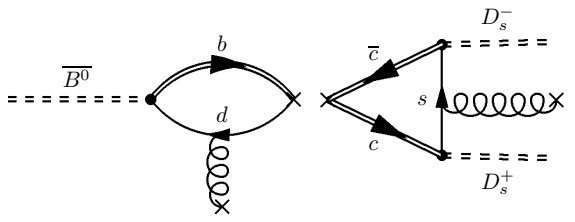

Figure 4. Non-factorizable contribution for $\overline{B^{0}} \rightarrow$ $D_{s}^{+} D_{s}^{-}$through the annihilation mechanism with additional soft gluon emision. The wavy lines represent soft gluons ending in vacuum to make gluon condensates.

merically $\rho$ is of order one [2])

Performing the bosonization of the $\mathrm{HL} \chi \mathrm{QM}$, one encounters divergent loop integrals which will in general be quadratic, linear and logarithmic divergent 2. The quadratic and logarithmic integrals are related to the quark condensate and the gluon condensate respectively 25]. The linearly divergent integral is related to $g_{\mathcal{A}}$.

The gluon condensate amplitudes can be written, within the framework presented in the previous section, in a quasi-factorized way as a product of matrix elements of colored currents: as visualized in Fig. 4 The left part in Fig. 4 gives us the bosonized colored current:

$$
\begin{array}{r}
\left(\overline{q_{L}} t^{a} \gamma^{\alpha} Q_{v_{b}}^{(+)}\right)_{1 G} \longrightarrow-\frac{G_{H} g_{s}}{64 \pi} G_{\mu \nu}^{a} \\
\times \operatorname{Tr}\left[\xi^{\dagger} \gamma^{\alpha} L H_{b}^{(+)}\left(\sigma^{\mu \nu}-F\left\{\sigma^{\mu \nu}, \gamma \cdot v_{b}\right\}\right)\right]
\end{array}
$$

where $G_{\mu \nu}^{a}$ is the octet gluon tensor, and $F \equiv$ $2 \pi f^{2} /\left(m^{2} N_{c}\right)$ is a dimensionless quantity of the order $1 / 3$. The symbol $\{$,$\} denotes the anti-$ commutator. For the creation of a $D \bar{D}$ pair in the right part of Fig. 4 the bosonization of the colored current $\left({\overline{Q_{v_{c}}^{(+)}}}^{a} \gamma^{\alpha} L Q_{\bar{v}}^{(-)}\right)_{1 G}$ is bosonized similarly to (15), but involves $H_{c}^{(+)}$and $H_{\bar{c}}^{(-)}$.

Multiplying the two colored currents and using the replacement:

$g_{s}^{2} G_{\mu \nu}^{a} G_{\alpha \beta}^{a} \rightarrow 4 \pi^{2}\left\langle\frac{\alpha_{s}}{\pi} G^{2}\right\rangle \frac{1}{12}\left(g_{\mu \alpha} g_{\nu \beta}-g_{\mu \beta} g_{\nu \alpha}\right)(16)$ we obtain a bosonized effective Lagrangian which is $1 / N_{c}$ suppressed compared to the factorized contributions. This effective Lagrangian correspond to a certain linear combination of a priori possible $1 / N_{c}$ suppressed terms at tree level (in the chiral perturbation theory sense).

\section{RESULTS}

Summing the chiral loops, the gluon condensate contributions and the annihilation contributions (for vectors in the final state), we obtain, for chiral loops regularized in the MS-bar scheme, the branching ratios: $B R\left(\bar{B}^{0} \rightarrow D_{s}^{+} D_{s}^{-}\right) \simeq$ $4.1 \times 10^{-4}, B R\left(\bar{B}^{0} \rightarrow D_{s}^{+*} D_{s}^{-}\right) \simeq 2.8 \times 10^{-4}$, $B R\left(\bar{B}^{0} \rightarrow D_{s}^{+} D_{s}^{-*}\right) \simeq 3.2 \times 10^{-4}$, and $B R\left(\bar{B}^{0} \rightarrow\right.$ $\left.D_{s}^{+*} D_{s}^{-*}\right) \simeq(20-50) \times 10^{-4}$. The masses of $D_{s}$ and $D_{s}^{*}$ are equal in the formal $m_{c} \rightarrow \infty$ limit. Especially the last branching ratio are sensitive to $1 / m_{c}$ corrections, which should be included in a future step of the calculations, like in $[\underline{3}$. The numbers above should therefore be considered as preliminary estimates. For further details, see [6], where results for $\overline{B_{s}^{0}} \rightarrow D^{(*)} \overline{D^{(*)}}$ will also be presented.

\section{REFERENCES}

1. J.O. Eeg, S. Fajfer, A. Hiorth, Phys. Lett. B 570 (2003) 46-52.

2. See: A. Hiorth and J. O. Eeg, Phys. Rev. D 66 (2002) 074001, and references therein.

3. A. Hiorth and J. O. Eeg, Eur.Phys.J.direct C30 (2003) 006.

4. B. Grinstein, W. Kilian, T. Mannel, and M.B. Wise, Nucl. Phys. B363 (1991) 19.

R. Fleischer, Nucl. Phys. B 412 (1994) 201.

5. See for example: S. Bertolini, J.O. Eeg and M. Fabbrichesi, Nucl. Phys. B449 (1995) 197, V. Antonelli, S. Bertolini, J.O. Eeg, M. Fabbrichesi and E.I. Lashin, Nucl. Phys. B469 (1996) 143, and references therein.

6. J.O. Eeg, S. Fajfer, and A. Prapotnik, in preparation. 